\title{
3 | 2018
}

18. Jahrgang

15. Juni 2018

S. $93-136$

PVSt 10439

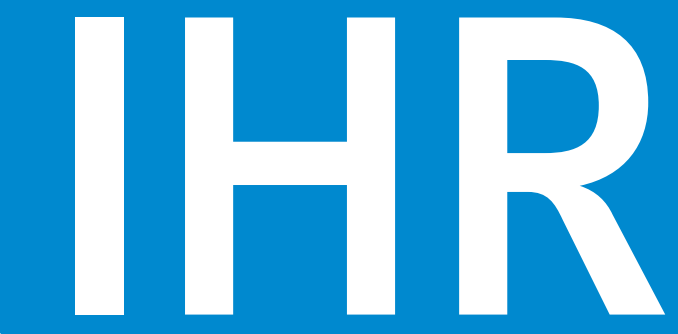

Zeitschrift für das Recht des

\section{Internationales Handelsrecht}

internationalen Warenkaufs und Warenvertriebs

Herausgegeben von: RA Prof. Dr. Rolf Herber · RiOLG Prof. Dr. Ulrich Magnus ·

RA Prof. Dr. Burghard Piltz · RA Dr. Karl-Heinz Thume

internationales-handelsrecht.net

gemeinsam mit: MDgtin. Prof. Dr. G. Beate Czerwenka - RA Dr. Tobias Eckardt · Prof. Dr. Franco

Ferrari · Prof. Dr. Christiana Fountoulakis · RA Prof. Dr. F. Christian Genzow · RA Dr. Christian

Groß · Prof. Dr. Peter Huber · RA Prof. Dr. Stefan Kröll · Prof. Dr. Brigitta Lurger · Prof. Dr. Peter

Mankowski · Prof. Dr. Ingo Saenger · Prof. Dr. Ingeborg Schwenzer

Aufsätze > Kemal Senocak / Ali Yarayan - Sachmängelhaftung des Verkäufers

beim Handelskauf nach türkischem Recht

Ramazan Uslubas - Die Rang- und Vorrangstellung des CISG nach

türkischem Recht

Entscheidungen > Hongkong ist kein Vertragsstaat des CISG (OLG Koblenz, Urt. v.

10.8.2015 - 12 U 580/11) m. Anm. Burghard Piltz

Mängelrüge und Beweismaß unter CISG (Schweiz: KG Will,

Entscheid v. 13.4.2016 - W.2015.33-WI2ZE-DWE) m. Anm. Christiana

Fountoulakis

CISG verdrängt einzelstaatliches Kollisionsrecht und parol evidence rule (US District Court for the ND of Illinois: Eastern Division, Memo-

randum Opinion and Order v. 31.3.2017 - 15 C 2207)

Umfang der Untersuchungsobliegenheit nach $§ 377$ Abs. 1 HGB und deren Konkretisierung in AGB (BGH, Urt. v. 6.12.2017 - VIII ZR 246/16,

ECLI:DE:BGH:2017:061217UVIIIZR246.16.0) 116

Handelsvertreterausgleichsanspruch des Kommissionsagenten

(OLG München, Urt. v. 20.12.2017 - 7 U 260/17)

Gerichtsstandsklausel in AGB eines Vertriebsvertrages (EuGH, Urt. v.

8.3.2018 - Rs. C-64/17, ECLI:EU:C:2018:173) 


\section{Führt Sie sicher durch das Datenschutzrecht.}

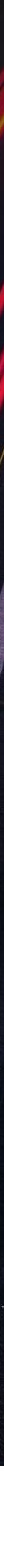

Plath DSGVO / BDSG

Kommentar zu DSGVO, BDSG und den Datenschutzbestimmungen des TMG und TKG. Herausgegeben von RA Dr. Kai-Uwe Plath und bearbeitet von 14 Autoren. 3., neu bearbeitete Auflage 2018, ca. 1.700 Seiten, gbd., 159,- $€$. ISBN 978-3-504-56075-1
Die dritte Auflage des Plath enthält alles, was Sie brauchen, um Ihr Unternehmen datenschutzrechtlich auf Touren zu bringen. Geblieben ist das bewährte Konzept, DSGVO und BDSG vernetzt in einem Werk zu kommentieren. Pragmatisch, lösungsorientiert und bewusst unternehmensbezogen behandelt es die zentralen Datenschutzthemen der betrieblichen Praxis. Wo das Gesetz Auslegungsspielräume lässt, bieten die Autoren Lösungsansätze für praxisnahe Fallgruppen. Dabei zeigen sie sich gewohnt meinungsfreudig und ordnen auch abweichende Ansichten zuverlässig ein.

Leseprobe und Infos unter www.otto-schmidt.de/bdsg3 